\title{
The effect of dietary antioxidant on the COPD risk: the community-based KoGES (Ansan- Anseong) cohort
}

This article was published in the following Dove Press journal:

International Journal of COPD

8 October 2015

Number of times this article has been viewed

\section{Pankaj Joshi ${ }^{1,2}$ \\ Woo Jin $\mathrm{Kim}^{3,4}$ \\ Sang-Ah Lee ${ }^{1-3}$}

'Department of Preventive Medicine, ${ }^{2}$ BIT Medical Convergence Graduate Program, ${ }^{3}$ Department of Internal Medicine, ${ }^{4}$ Environmental Health Center, Kangwon National University School of Medicine, Chuncheon-si, Gangwon-do, South Korea
Correspondence: Sang-Ah Lee Department of Preventive Medicine, Kangwon National University School of Medicine, I Kangwondaehak-gil, Chuncheon-si, Gangwon-do 200-70I, South Korea

Tel +82332508871

Fax +82332508809

Email sangahlee@kangwon.ac.kr
Background: Dietary antioxidants have been suggested to have protective role against chronic obstructive pulmonary disease (COPD), but few prospective studies examined this relationship. The prospective study was conducted to evaluate the effect of dietary antioxidants on COPD risk and lung function in the Korean population.

Methods: The data were collected from the community-based Korean Genome Epidemiology Study (KoGES) cohort. To diagnose COPD, forced expiratory volume $\left(\mathrm{FEV}_{1}\right)$ and forced vital capacity (FVC) were measured by spirometry. The dietary intake of antioxidant vitamins was estimated from validated Food-Frequency Questionnaire. For the analysis, 325 COPD patients and 6,781 at risk subjects were selected from the cohort of 10,038 subjects. Multiple logistic regression models were used to examine the odds ratio $(\mathrm{OR})$ after adjusting for age, sex, marital status, income, history of asthma, history of tuberculosis, and smoking.

Results: The risk of COPD was positively associated with aging, low education, low household income, lower body mass index, and cigarette smoking. The risk of COPD decreased with increase in the dietary vitamin $\mathrm{C}\left(\mathrm{OR}_{\mathrm{Q} 1 \text { vs } Q 5}=0.66, P_{\text {trend }}=0.03\right)$ and vitamin $\mathrm{E}\left(\mathrm{OR}_{\mathrm{Q} 1 \text { vs } \mathrm{Q} 5}=0.56\right.$, $\left.P_{\text {trend }}=0.05\right)$ intake, predominantly, in men $\left(P_{\text {trend }}=0.01\right.$ and 0.05 for vitamins $\mathrm{C}$ and $\mathrm{E}$, respectively). In addition, the lung function was significantly improved with increase in vitamins $\mathrm{C}$ $\left(\mathrm{FEV}_{1}, P=0.04\right.$; FVC, $\left.P=0.03\right)$ and $\mathrm{E}\left(\mathrm{FEV}_{1}, P=0.03\right.$; FVC, $\left.P=0.04\right)$ intake. No statistically significant interactions were observed between smoking and vitamin $\mathrm{C}$ or $\mathrm{E}$ intake in relation to COPD risk among men.

Conclusion: Our results suggest the independent beneficial effect of antioxidants, particularly vitamins $\mathrm{C}$ and $\mathrm{E}$, on COPD risk and lung function in men.

Keywords: chronic obstructive pulmonary disease (COPD), antioxidant vitamins intake, smoking, lung function, the combined effect of smoking and antioxidant vitamins

\section{Introduction}

Chronic obstructive pulmonary disease (COPD) is a preventable public health problem, with high prevalence in countries having higher rate of cigarette smoking. ${ }^{1} \mathrm{COPD}$ mortality has been decreasing in the Asia-Pacific region, but has been increasing in South Korea. ${ }^{2}$ Cigarette smoke increases the burden of oxidants on the lungs and may activate inflammatory response that induces reactive oxygen species (ROS). High level of ROS in the lungs increases lipid peroxidation, activation of nuclear factor-kappa B (NFKB), signal transduction, chromatin remodeling, and gene expression of proinflammatory mediators, which cause inflammation in COPD patients. ${ }^{3}$

Dietary antioxidants, mainly vitamins $\mathrm{C}$ and $\mathrm{E}$, are found responsible for antioxidant defenses in the lungs. ${ }^{4}$ Vitamin $\mathrm{C}$ scavenges superoxide radicals and protects against oxidative damage, and vitamin E breaks the lipid peroxidation chain and functions as 
the primary defense against oxidative damage. ${ }^{5}$ A prospective study showed that diet rich in fruits and vegetables may reduce the risk of COPD. ${ }^{6}$ Results of prospective study demonstrated a statistically significant association of dietary vitamin $\mathrm{C}$ with the level of pulmonary function ${ }^{7}$ and protective effect of vitamin $\mathrm{E}$ intake on COPD risk. ${ }^{8}$ In another prospective study, higher levels of vitamins $\mathrm{C}$ and $\mathrm{E}$ in serum and diet were found associated with an increase in forced expiratory volume $\left(\mathrm{FEV}_{1}\right)$ and forced vital capacity (FVC) in healthy subjects. ${ }^{9}$ These findings were supported by a randomized controlled trial that suggested an association of a dietary shift to higher intake of antioxidants food with improvement in lung function. ${ }^{10}$ Furthermore, Tsiligianni and van der Molen ${ }^{11}$ reported an effect of those vitamins with lung function in the COPD patients. In addition, several cross-sectional studies suggested the protection of airways by dietary antioxidants against oxidant-mediated damage of lungs leading to COPD. ${ }^{12,13}$ Although several epidemiological studies focused on overall diet and its association with COPD risk and lung function, we wanted to further explore the relationship of the dietary antioxidant vitamins with the risk of COPD and lung function prospectively.

Thus, this paper aimed at investigating the association of the antioxidant vitamin intake with the risk of COPD and lung function and at evaluating the combined effect of smoking and the selected antioxidant vitamin intake on the COPD risk using prospective study.

\section{Methods}

\section{Study population}

Detailed information on the study design and procedures is available in a previous report. ${ }^{14}$ Briefly, this study is was conducted on the community-based Korean Genome and Epidemiology Study (KoGES) cohort and included subjects from the Ansan (urban) and Anseong (rural) areas for prospective investigation. The baseline study was conducted from May 2001 to February 2003. First and second follow-up was conducted from April 2003 to February 2005 and February 2005 to November 2006, respectively. Study procedures were in accordance with institutional guidelines and were approved by the Korean National Institute of Health institutional review committee. Each participant signed an informed consent form before the baseline health examination and during every visit, approved by Human Subjects Committee.

For the analysis, we excluded participants below 40 and above 69 years of age $(n=39)$, participants who did not complete questions related to COPD information at baseline $(n=247)$, and participants who had no knowledge of dietary information or reported inacceptable energy intake on the health examination $(n=625)$. After exclusion, 9,127 subjects were taken as study population. Since the outcome measure for this study was new cases of COPD, participants who were determined to have COPD at baseline ( $\mathrm{n}=867)$, who did not report the COPD information of first and second follow-up $(\mathrm{n}=1,410)$, and who reported the COPD $\left(\mathrm{FEV}_{1} / \mathrm{FVC}<0.70\right)$ at first follow-up but were normal at second follow-up $(n=69)$ were also excluded. After the additional exclusions, 6,781 participants ( $45.7 \%$ men and $54.3 \%$ women) entered the analysis for the first 2-year follow-up. Those who were subjects of first follow-up and who were not cases of COPD remained in the analysis, $75 \%$ of the participants at the baseline, for the second 2-year follow-up (Figure 1).

Lung function was measured by spirometry at every visit (at baseline, in first and second follow-up), VMAX2130 from Sensormedics Corporation, Yorba, CA, USA. A GOLD guideline for the assessment for COPD was adopted from the American Thoracic Society. ${ }^{1}$ A prebronchodilator $\mathrm{FEV}_{1} /$ FVC ratio $<0.70$ was defined as COPD in subjects.

\section{Dietary assessment}

The validation and reproducibility of the Food-Frequency Questionnaire (FFQ) is described in detail elsewhere, ${ }^{15}$ and dietary information was obtained using a semi-FFQ. Participants were asked to complete the FFQ with inquiries about information of the average consumption frequency and serving size for 103 food items. The frequency of servings was classified into nine categories: never or seldom, once a month, 2-3 times a month, 1-2 times a week, 3-4 times a week, 5-6 times a week, once a day, twice a day, or 3 times or more every day. For the food items with different seasonal availability, the participants were requested to answer on how long they ate among the four categories: 3, 6, 9, and 12 months. The portion size, classified as small, medium, and large, was determined depending on the median value of each food determined from the 24 hours recall data obtained from the Korea Health and Nutrition Examination Survey in 1998. The nutrient intake values were obtained by cumulative percent contribution, which is described in detail elsewhere. ${ }^{16}$ The dietary intake information was collected at baseline.

\section{Statistical analysis}

Descriptive statistics were used to summarize participant demographic and lifestyle factors, and logistic regression was used to estimate their effect on the COPD risk for COPD patients and subjects at risk. Study participants were classified into five categories by quintile of the intake of 


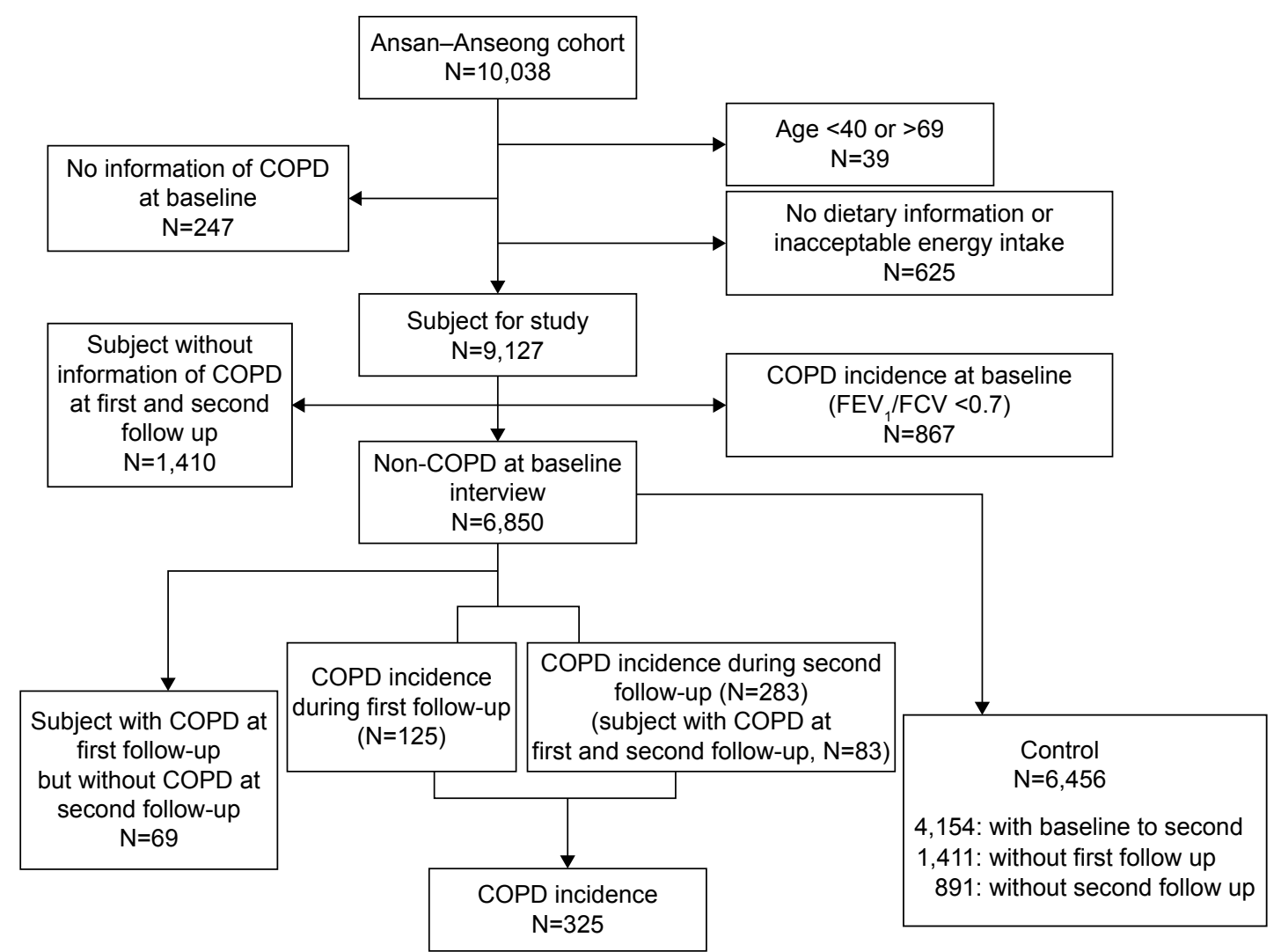

Figure I The flow diagram of selection of study population from Ansan and Anseong cohort.

each nutrient. The lowest quintile served as the reference group in the analyses. Odds ratio (OR) and $95 \%$ confidence interval (CIs) summarizing the association between COPD risk and nutrient intake were calculated by using pooled logistic regression after adjustment for age, sex, marriage status, body mass index (BMI), energy, history of asthma and tuberculosis, and cigarette smoking. Multiple linear regression models were employed to determine the associations between the intake of vitamin C (increased $100 \mathrm{mg}$ ) and vitamin $\mathrm{E}$ (increased $5 \mathrm{mg}$ ) and $\mathrm{FEV}_{1}$ and $\mathrm{FVC}$. For the combined analysis between vitamins $\mathrm{C}$ or $\mathrm{E}$ intake and smoking on COPD risk, tests for interaction were performed by comparing the model with and without interaction terms by using likelihood ratio test. The term statistically significant refers to a $P$-value $<0.05$. All statistical tests were based on two-sided probability. All the statistical analyses were performed using SAS statistical software (version 9.2, SAS Institute Inc., Cary, NC, USA).

We stratified the analysis according to sex, as one published paper reported that gender influences COPD. ${ }^{17}$ As $\mathrm{BMI}$ is the important predictor of the lung function, ${ }^{18}$ low $\mathrm{BMI}$ is highly related to $\mathrm{COPD},{ }^{19}$ and there is a strong interrelation between diet and BMI, we adjusted for BMI in the regression model.

\section{Results}

The general characteristics and lifestyle factors of the study population is described in Table 1. Among the subjects at risk, 325 COPD patients were found during first and second follow-up. COPD patients were slightly older than subjects at risk, with a mean age of 56.7 years as compared to 51.0 years.

The effect of general characteristics and lifestyle factors on the risk of COPD is listed in Table 2. The risk of COPD increased with age $\left(P_{\text {trend }}<0.01\right)$, was decreased in females $(\mathrm{OR}=0.35)$, and was increased in whose marital status is single $(\mathrm{OR}=1.43)$. Subjects with the history of asthma (OR $=3.68)$ and tuberculosis $(\mathrm{OR}=1.84)$ were likely to be diagnosed with the COPD, but subjects with the history of chronic respiratory diseases were not. Cigarette smoking was associated with the risk of COPD (OR $=3.35$ for current smoker and OR $=2.20$ for former smoker), but no association was observed for smoking pack years and alcohol drinking. Higher BMI was significantly associated with decreased risk of COPD. 
Table I The general characteristics and lifestyle factors of study population

\begin{tabular}{|c|c|c|}
\hline & $\begin{array}{l}\text { Subjects at risk } \\
(n=6,78 I)\end{array}$ & $\begin{array}{l}\text { COPD } \\
(n=325)\end{array}$ \\
\hline & $51.0 \pm 8.45$ & $56.7 \pm 9.02$ \\
\hline \multicolumn{3}{|l|}{ Age } \\
\hline $40-44$ & $1,966(29.0)$ & $52(16.0)$ \\
\hline $45-49$ & $1,548(22.8)$ & $36(11.1)$ \\
\hline $50-54$ & $898(13.2)$ & $32(9.90)$ \\
\hline $55-59$ & $872(12.9)$ & 51 (I5.7) \\
\hline $60-64$ & $815(12.0)$ & $72(22.2)$ \\
\hline $65-69$ & $682(10.1)$ & $82(25.2)$ \\
\hline \multicolumn{3}{|l|}{ Sex } \\
\hline Male & $3,098(44.0)$ & $255(78.5)$ \\
\hline Female & $3,683(54.3)$ & $70(21.5)$ \\
\hline \multicolumn{3}{|l|}{ BMI } \\
\hline$<25 \mathrm{BMI}$ & $3,738(55.12)$ & $219(67.4)$ \\
\hline $25 \leq \mathrm{BMI}$ & $3,043(44.9)$ & $106(32.6)$ \\
\hline \multicolumn{3}{|l|}{ Job } \\
\hline Housekeeper & $1,966(29.5)$ & $30(9.30)$ \\
\hline Office worker & $336(5.00)$ & $13(4.00)$ \\
\hline Farmer & I,632 (24.I) & $136(42.1)$ \\
\hline Private business & $1,040(15.4)$ & $42(13.0)$ \\
\hline Sales work & $96(1.40)$ & $0(0.00)$ \\
\hline Blue-collar job & $390(5.80)$ & $13(4.00)$ \\
\hline Professional & $295(4.40)$ & $16(5.00)$ \\
\hline Others & $976(14.4)$ & $73(22.6)$ \\
\hline \multicolumn{3}{|l|}{ Education obtained } \\
\hline Elementary & $2,049(30.3)$ & $122(37.8)$ \\
\hline Middle school & I,497 (22.2) & $66(20.4)$ \\
\hline High school & $2,185(32.4)$ & $95(29.4)$ \\
\hline More than college & $1,022(15.1)$ & $40(12.4)$ \\
\hline \multicolumn{3}{|l|}{ Marriage status } \\
\hline In a relationship & $6,138(90.5)$ & $287(88.3)$ \\
\hline Single & $643(9.50)$ & 38 (11.7) \\
\hline \multicolumn{3}{|l|}{ Type of house } \\
\hline Single house & $3,407(50.3)$ & 207 (63.9) \\
\hline Apartment & $2,105(31.1)$ & $63(19.4)$ \\
\hline Multiplex housing & $1,176(17.4)$ & $48(14.8)$ \\
\hline Others & $87(1.30)$ & $6(1.90)$ \\
\hline \multicolumn{3}{|l|}{ Income (USD) } \\
\hline$<500$ & $1,082(16.2)$ & 81 (25.2) \\
\hline $500-990$ & $989(14.8)$ & $62(19.3)$ \\
\hline $\mid, 000-1,490$ & $1,047(15.6)$ & $54(16.8)$ \\
\hline $1,500-1,990$ & $970(14.5)$ & $40(12.4)$ \\
\hline $2,000-2,990$ & $1,314(19.6)$ & 44 (13.7) \\
\hline$\geq 3,000$ & $1,292(19.3)$ & $41(12.7)$ \\
\hline \multicolumn{3}{|l|}{ Self-rated health } \\
\hline Very bad & $222(3.30)$ & $13(4.00)$ \\
\hline Bad & $1,936(28.6)$ & $90(27.8)$ \\
\hline Normal & $2,472(36.5)$ & $114(35.2)$ \\
\hline Good & $2,036(30.1)$ & $102(31.5)$ \\
\hline Very good & $100(1.50)$ & $5(1.50)$ \\
\hline \multicolumn{3}{|c|}{ Physician-reported comorbidity (yes) } \\
\hline Asthma & $103(1.50)$ & II (3.40) \\
\hline Chronic respiratory & $36(0.50)$ & $5(1.60)$ \\
\hline Tuberculosis & $314(4.60)$ & $27(8.40)$ \\
\hline \multicolumn{3}{|l|}{ Cigarette smoker } \\
\hline Never & $4,145(61.6)$ & $87(26.9)$ \\
\hline Formerly & $\mathrm{I}, 045(15.5)$ & $82(25.3)$ \\
\hline Currently & $1,540(22.9)$ & $155(47.8)$ \\
\hline
\end{tabular}

Table I (Continued)

\begin{tabular}{lll}
\hline & $\begin{array}{l}\text { Subjects at risk } \\
(\mathbf{n}=\mathbf{6 , 7 8} \mathbf{)})\end{array}$ & $\begin{array}{l}\text { COPD } \\
(\mathbf{n}=\mathbf{3 2 5})\end{array}$ \\
\hline $\begin{array}{l}\text { Smoking pack year } \\
<\text { I4 PYR }\end{array}$ & $5,171(76.3)$ & $158(48.6)$ \\
$\quad \begin{array}{l}\text { I4 PYR } \\
\text { Alcohol drinker }\end{array}$ & $1,610(23.7)$ & $167(51.4)$ \\
$\quad \begin{array}{l}\text { Never } \\
\text { Formerly }\end{array}$ & $3,168(46.9)$ & $101(31.2)$ \\
$\quad$ Currently & $372(5.50)$ & $29(9.00)$ \\
Exercise & $3,219(47.6)$ & $194(59.9)$ \\
$\quad$ No & & $243(74.8)$ \\
Yes & $4,776(70.4)$ & $82(25.3)$ \\
\hline
\end{tabular}

Abbreviations: BMI, body mass index; PYR, pack year.

Table 2 The effect of general characteristics and lifestyle factors on the risk of COPD

\begin{tabular}{|c|c|c|}
\hline & cOR $(95 \% \mathrm{CI})$ & $\operatorname{adjOR}(95 \% \mathrm{Cl})^{\mathrm{a}}$ \\
\hline \multicolumn{3}{|l|}{ Age, in years } \\
\hline $40-44$ & Ref & Ref \\
\hline $45-49$ & $0.88(0.57-1.35)$ & $0.79(0.5 \mathrm{I}-\mathrm{I} .23)$ \\
\hline $50-54$ & $1.36(0.87-2.13)$ & $1.25(0.78-2.01)$ \\
\hline $55-59$ & $2.29(1.54-3.39)$ & $2.38(1.53-3.70)$ \\
\hline $60-64$ & $3.57(2.47-5.15)$ & $4.01(2.59-6.21)$ \\
\hline \multirow[t]{2}{*}{$65-69$} & $5.03(3.5|-7.2|)$ & $5.08(3.22-8.03)$ \\
\hline & & $P_{\text {trend }}<0.01$ \\
\hline \multicolumn{3}{|l|}{ Sex } \\
\hline Male & Ref & Ref \\
\hline Female & $0.22(0.17-0.28)$ & $0.35(0.21-0.57)$ \\
\hline \multicolumn{3}{|l|}{ BMI } \\
\hline$<25 \mathrm{BMI}$ & Ref & Ref \\
\hline \multirow[t]{2}{*}{$25 \leq \mathrm{BMI}$} & $0.58(0.46-0.74)$ & $0.71(0.55-0.91)$ \\
\hline & & $P_{\text {trend }}=0.01$ \\
\hline \multicolumn{3}{|l|}{ Job } \\
\hline Housekeeper & $0.35(0.19-0.65)$ & $0.77(0.36-1.62)$ \\
\hline Office worker & Ref & Ref \\
\hline Farmer & $2.07(1.20-3.57)$ & $0.98(0.52-1.84)$ \\
\hline Private business & $0.96(0.52-1.75)$ & $0.97(0.52-I .8 I)$ \\
\hline Sales work & - & - \\
\hline Blue-collar job & $0.78(0.37-1.67)$ & $1.06(0.48-2.34)$ \\
\hline Professional & $1.30(0.63-2.68)$ & $\mathrm{I} .19(0.56-2.5 \mathrm{I})$ \\
\hline Others & $1.84(1.04-3.25)$ & $1.16(0.63-2.13)$ \\
\hline \multicolumn{3}{|l|}{ Education obtained } \\
\hline Elementary & Ref & Ref \\
\hline Middle school & $0.73(0.54-0.99)$ & $0.95(0.67-1.36)$ \\
\hline High school & $0.72(0.54-0.94)$ & $0.95(0.66-1.36)$ \\
\hline \multirow[t]{2}{*}{ More than college } & $0.64(0.45-0.92)$ & $0.71(0.45-1.14)$ \\
\hline & & $P_{\text {trend }}=0.27$ \\
\hline \multicolumn{3}{|l|}{ Marriage status } \\
\hline In a relationship & Ref & Ref \\
\hline Single & $1.28(0.90-1.82)$ & $1.43(0.96-2.13)$ \\
\hline \multicolumn{3}{|l|}{ Type of house } \\
\hline Single house & Ref & Ref \\
\hline Apartment & $0.48(0.36-0.63)$ & $0.77(0.53-1.12)$ \\
\hline Multiplex housing & $0.66(0.48-0.90)$ & $0.93(0.63-1.37)$ \\
\hline Others & $1.14(0.49-2.65)$ & $1.29(0.53-3.18)$ \\
\hline
\end{tabular}


Table 2 (Continued)

\begin{tabular}{|c|c|c|}
\hline & cOR $(95 \% \mathrm{Cl})$ & $\overline{\operatorname{adjOR}(95 \% \mathrm{Cl})^{\mathrm{a}}}$ \\
\hline \multicolumn{3}{|l|}{ Income (USD) } \\
\hline$<500$ & Ref & Ref \\
\hline $500-990$ & $0.86(0.62-1.21)$ & $0.88(0.61-1.28)$ \\
\hline$I, 000-1,490$ & $0.70(0.50-1.00)$ & $0.90(0.60-1.34)$ \\
\hline $1,500-1,990$ & $0.56(0.38-0.82)$ & $0.87(0.55-1.38)$ \\
\hline $2,000-2,990$ & $0.45(0.31-0.65)$ & $0.83(0.52-1.33)$ \\
\hline \multirow[t]{2}{*}{$\geq 3,000$} & $0.42(0.29-0.62)$ & $0.81(0.49-1.34)$ \\
\hline & & $P_{\text {trend }}=0.10$ \\
\hline \multicolumn{3}{|l|}{ Self-rated health } \\
\hline Very bad & Ref & Ref \\
\hline Bad & $0.78(0.44-\mathrm{I} .40)$ & $0.81(0.43-1.49)$ \\
\hline Normal & $0.77(0.44-1.37)$ & $0.85(0.46-1.58)$ \\
\hline Good & $0.84(0.47-1.49)$ & $0.91(0.49-1.7 I)$ \\
\hline \multirow[t]{2}{*}{ Very good } & $0.84(0.29-2.39)$ & $0.85(0.28-2.55)$ \\
\hline & & $P_{\text {trend }}=0.56$ \\
\hline \multicolumn{3}{|c|}{ Physician-reported comorbidity (yes) } \\
\hline Asthma & $2.42(1.28-4.58)$ & $3.68(1.84-7.38)$ \\
\hline Chronic respiratory & $3.24(1.25-8.38)$ & $1.38(0.50-3.82)$ \\
\hline Tuberculosis & $1.95(1.29-2.94)$ & $1.84(1.18-2.87)$ \\
\hline \multicolumn{3}{|l|}{ Cigarette smoker } \\
\hline Never & Ref & Ref \\
\hline Formerly & $3.98(2.92-5.4 I)$ & $2.20(1.45-3.35)$ \\
\hline \multirow[t]{2}{*}{ Currently } & $5.22(3.99-6.83)$ & $3.35(2.28-4.92)$ \\
\hline & & $P_{\text {trend }}<0.01$ \\
\hline \multicolumn{3}{|l|}{ Smoking pack year } \\
\hline$<14$ PYR & Ref & Ref \\
\hline$\geq 14$ PYR & $3.67(2.93-4.60)$ & $1.14(0.84-1.56)$ \\
\hline \multicolumn{3}{|l|}{ Alcohol drinker } \\
\hline Never & Ref & Ref \\
\hline Formerly & $0.51(0.40-0.66)$ & I.II (0.82-I.52) \\
\hline \multirow[t]{2}{*}{ Currently } & $1.32(0.88-1.98)$ & $1.06(0.68-1.64)$ \\
\hline & & $P_{\text {trend }}=0.52$ \\
\hline \multicolumn{3}{|l|}{ Exercise } \\
\hline No & Ref & Ref \\
\hline Yes & $0.80(0.62-1.03)$ & $0.94(0.7|-| .24)$ \\
\hline
\end{tabular}

Notes: adjusted for age, sex, marriage status, BMI, energy, history of asthma and tuberculosis, and cigarette smoking.

Abbreviations: BMI, body mass index; PYR, pack year; cOR, crude odds ratio; adjOR, adjusted odds ratio; $\mathrm{Cl}$, confidence interval.

The association of daily nutrient intake with the risk of COPD is summarized in Table 3. Daily intake of total energy, protein, fat, carbohydrate, and fiber was not associated with risk of COPD. The higher the intake of retinol $\left(\mathrm{OR}_{\mathrm{Q} 1 \text { vs } \mathrm{Q} 5}=0.56, P_{\text {trend }}=0.05\right)$, vitamin $\mathrm{C}\left(\mathrm{OR}_{\mathrm{Q} 1 \mathrm{vs} \mathrm{Q} 5}=0.66\right.$, $\left.P_{\text {trend }}=0.03\right)$, and vitamin $\mathrm{E}\left(\mathrm{OR}_{\mathrm{Q} 1 \text { vs Q5 }}=0.56, P_{\text {trend }}=0.05\right)$, the more the decrease in COPD risk. No association was observed between other vitamin intake and COPD risk.

Table 4 lists the association between nutrient intake and COPD risk after stratification by sex. The adjusted risk of COPD decreased in men who belonged to the highest quintile of retinol $\left(\mathrm{OR}=0.46, P_{\text {trend }}=0.01\right)$ and vitamin $\mathrm{C}$ $\left(\mathrm{OR}=0.61, P_{\text {trend }}=0.01\right)$ intake when compared with lowest quintile for each antioxidant vitamin; however, the risk was not associated vitamin E intake. By contrast, no association of selected antioxidant vitamin was observed on COPD risk for women, although the intake of vitamin $\mathrm{E}$ was lower in COPD women than in women at risk.

Table 5 lists the association of $\mathrm{FEV}_{1}, \mathrm{FVC}$, and $\mathrm{FEV}_{1} / \mathrm{FVC}$ (index of lung function) with increased intake of vitamins $\mathrm{C}$ and E. An increase in vitamin C intake of $100 \mathrm{mg} / \mathrm{d}$ was associated with an increase of $17.1 \mathrm{~mL}$ in $\operatorname{FEV}_{1}(P=0.03)$ and $24.1 \mathrm{~mL}$ in $\mathrm{FVC}(P=0.01)$. In addition, an increase in intake of vitamin $\mathrm{E}$ of $5 \mathrm{mg} / \mathrm{d}$ was associated with an increase of $1.64 \mathrm{~mL}$ in $\operatorname{FEV}_{1}(P=0.02)$ and $2.01 \mathrm{~mL}$ in $\mathrm{FVC}(P=0.03)$. No association was observed between increased intake of vitamins $\mathrm{C}$ and $\mathrm{E}$ and $\mathrm{FEV}_{1} / \mathrm{FVC}$ ratio.

The combined effect of smoking and dietary antioxidant vitamin $\mathrm{C}$ or $\mathrm{E}$ intake on COPD risk among men is summarized in Table 6. The risk of COPD for current smokers with lowest intake of vitamins $\mathrm{C}$ or $\mathrm{E}$ increased by 2.96 -fold ( $95 \%$ $\mathrm{CI}=1.88-4.67)$ and 2.98 -fold (95\% CI $=1.78-4.97)$, respectively, greater than for noncurrent smokers with high intake of vitamins $\mathrm{C}$ or $\mathrm{E}$, although tests for an interaction effect were not significant. On the other hand, no association was observed between antioxidant vitamins $\mathrm{C}$ or $\mathrm{E}$ intake and COPD risk after stratification by smoking status: for noncurrent smoker, the ORs of vitamins $\mathrm{C}$ and $\mathrm{E}$ intake were 1.26 (95\% CI $=0.77-2.05$ ) and 1.48 (95\% CI $=0.84-2.59$ ), respectively; and for current smoker, the ORs were $1.21(95 \% \mathrm{CI}=0.78-1.89)$ and $0.96(95 \%$ $\mathrm{CI}=0.60-1.55)$, respectively (data not shown).

\section{Discussion}

We conducted a comprehensive study of the association between dietary antioxidant and COPD risk as well as lung function. The risk of COPD was positively associated with aging, low education, low household income, lower BMI, and cigarette smoking. For the intake of antioxidant vitamins, a negative association with COPD risk was observed in the intake of retinol, vitamins $\mathrm{C}$ and $\mathrm{E}$, predominantly in men. In addition, the protective effect of vitamins $\mathrm{C}$ and $\mathrm{E}$ on the lung function was observed in a dose-dependent manner; an increase in vitamins $\mathrm{C}$ and $\mathrm{E}$ intake was significantly associated with an increase in $\mathrm{FEV}_{1}$ and FVC. For the combined effect of smoking and the selected antioxidant vitamins on COPD risk among men, we observed that the current smoker with the lowest vitamin $\mathrm{C}$ or $\mathrm{E}$ intake had a higher relative risk than the noncurrent smoker with the highest intake of the vitamins $\mathrm{C}$ or $\mathrm{E}$, although we could not find a statistically significant effect of interaction.

Consistent with the findings of other studies, increased risk of COPD was seen in aged subjects ${ }^{20}$ and in males ${ }^{21}$ in 
Table 3 The association between daily nutrients intake and COPD risk

\begin{tabular}{|c|c|c|c|c|c|}
\hline & \multicolumn{4}{|l|}{ OR } & \multirow[t]{2}{*}{$P_{\text {for trend }}$} \\
\hline & Q2 & Q3 & Q4 & Q5 & \\
\hline \multicolumn{6}{|c|}{ Energy-source nutrients } \\
\hline Energy (kcal) & $0.77(0.52-1.13)$ & $0.77(0.52-1.13)$ & $1.03(0.70-1.50)$ & $1.00(0.68-1.47)$ & 0.53 \\
\hline Protein $(\mathrm{g})$ & $0.85(0.56-1.28)$ & $0.85(0.56-1.28)$ & I.0I (0.58-I.74) & $0.72(0.37-1.39)$ & 0.54 \\
\hline Fat $(\mathrm{g})$ & $0.86(0.58-1.29)$ & $0.86(0.58-1.29)$ & $0.87(0.54-1.38)$ & $0.76(0.44-1.33)$ & 0.44 \\
\hline Carbohydrate (g) & $0.91(0.60-1.37)$ & $0.91(0.60-1.37)$ & $0.84(0.44-1.63)$ & $0.76(0.34-1.70)$ & 0.53 \\
\hline Fiber (g) & $0.98(0.67-1.43)$ & $0.98(0.67-1.43)$ & $1.09(0.72-1.66)$ & $0.92(0.58-1.45)$ & 0.93 \\
\hline \multicolumn{6}{|l|}{ Antioxidant vitamins } \\
\hline Vitamin A (RE) & $1.16(0.80-1.69)$ & $1.12(0.75-1.65)$ & $0.92(0.60-1.40)$ & I.II (0.73-I.68) & 0.95 \\
\hline Retinol $(\mu \mathrm{g})$ & $0.78(0.54-I .12)$ & I.03 (0.7I-I.49) & $0.86(0.58-1.27)$ & $0.56(0.36-0.87)$ & 0.05 \\
\hline$\beta$-carotene $(\mu \mathrm{g})$ & $0.88(0.61-1.29)$ & $0.95(0.65-1.39)$ & $0.88(0.60-1.3 \mathrm{I})$ & $0.97(0.66-1.44)$ & 0.92 \\
\hline Vitamin C (mg) & $0.78(0.55-1.11)$ & $0.79(0.55-1.13)$ & $0.65(0.44-0.96)$ & $0.66(0.43-1.01)$ & 0.03 \\
\hline Folate $(\mu \mathrm{g})$ & $0.82(0.56-1.19)$ & $0.82(0.56-1.21)$ & I.07 (0.72-I.58) & $0.7 \mathrm{I}(0.46-\mathrm{I} . \mathrm{II})$ & 0.48 \\
\hline Vitamin E (mg) & $0.91(0.63-1.32)$ & $0.63(0.4 I-0.96)$ & $0.85(0.55-1.33)$ & $0.56(0.34-0.94)$ & 0.05 \\
\hline \multicolumn{6}{|l|}{ Other vitamins } \\
\hline Vitamin $B_{1}(m g)$ & $1.05(0.7 \mathrm{I}-\mathrm{I} .57)$ & $1.13(0.72-1.75)$ & $1.13(0.72-1.75)$ & $0.95(0.52-1.74)$ & 0.78 \\
\hline Vitamin $B_{2}(m g)$ & $0.76(0.5 I-I .12)$ & I.0I (0.67-I.5I) & I.0I (0.67-I.5I) & $0.58(0.34-1.00)$ & 0.12 \\
\hline Niacin (mg) & $0.92(0.62-1.36)$ & $0.79(0.50-1.23)$ & $0.79(0.50-1.23)$ & $0.77(0.43-1.39)$ & 0.60 \\
\hline Vitamin $B_{6}(\mathrm{mg})$ & $1.02(0.69-1.50)$ & $0.75(0.48-1.18)$ & $0.75(0.48-1.18)$ & $0.76(0.44-1.34)$ & 0.84 \\
\hline
\end{tabular}

Notes: QI is taken as reference; OR after adjusting for age, sex, marriage status, BMI, history of asthma and tuberculosis, energy, and cigarette smoking.

Abbreviations: OR, odds ratio; $Q$, quintile; RE, retinol equivalents; BMI, body mass index.

Table 4 The association between daily nutrients intake and COPD risk among men and women

\begin{tabular}{|c|c|c|c|c|c|}
\hline & \multicolumn{4}{|l|}{ OR } & \multirow[t]{2}{*}{$\boldsymbol{P}_{\text {for trend }}$} \\
\hline & Q2 & Q3 & Q4 & Q5 & \\
\hline \multicolumn{6}{|l|}{ Men } \\
\hline \multicolumn{6}{|c|}{ Energy-source nutrients } \\
\hline Energy (kcal) & $0.73(0.45-1.18)$ & $1.06(0.68-1.65)$ & $1.06(0.65-1.59)$ & $0.91(0.58-1.43)$ & 0.77 \\
\hline Protein $(g)$ & $0.87(0.53-1.44)$ & $0.96(0.55-1.66)$ & I.0I (0.53-1.93) & $0.66(0.30-1.42)$ & 0.45 \\
\hline Fat (g) & $0.98(0.60-1.6 \mathrm{I})$ & $1.18(0.72-1.96)$ & $0.80(0.45-1.40)$ & $0.70(0.37-1.35)$ & 0.19 \\
\hline Carbohydrate (g) & $1.18(0.72-1.95)$ & $0.94(0.5 \mathrm{I}-1.76)$ & $1.00(0.46-2.15)$ & $0.80(0.3 \mathrm{I}-2.03)$ & 0.53 \\
\hline Fiber $(g)$ & $1.06(0.68-1.64)$ & $0.89(0.56-1.42)$ & $0.97(0.60-1.60)$ & $0.80(0.47-1.35)$ & 0.37 \\
\hline \multicolumn{6}{|l|}{ Antioxidant vitamins } \\
\hline Vitamin A (RE) & I.3I (0.84-2.03) & I.08 (0.68-I.72) & $0.88(0.53-1.44)$ & $0.99(0.6 \mathrm{I}-\mathrm{I} .62)$ & 0.40 \\
\hline Retinol $(\mu \mathrm{g})$ & $0.81(0.53-1.22)$ & $0.94(0.6 \mathrm{I}-\mathrm{I} .46)$ & $0.79(0.50-1.25)$ & $0.46(0.28-0.78)$ & 0.01 \\
\hline$\beta$-carotene $(\mu \mathrm{g})$ & $1.02(0.66-1.58)$ & $0.98(0.63-1.54)$ & $0.83(0.52-1.33)$ & $0.92(0.58-1.45)$ & 0.48 \\
\hline Vitamin C (mg) & $0.83(0.56-1.23)$ & $0.68(0.45-1.04)$ & $0.56(0.36-0.89)$ & $0.6 \mathrm{I}(0.37-\mathrm{I} .0 \mathrm{I})$ & 0.01 \\
\hline Folate $(\mu g)$ & $0.82(0.53-1.27)$ & $0.83(0.53-1.30)$ & $0.86(0.54-1.37)$ & $0.58(0.34-0.97)$ & 0.09 \\
\hline Vitamin E (mg) & $0.89(0.58-1.38)$ & $0.53(0.32-0.87)$ & $0.78(0.46-1.3 \mathrm{I})$ & $0.54(0.31-0.98)$ & 0.06 \\
\hline \multicolumn{6}{|l|}{ Women } \\
\hline \multicolumn{6}{|c|}{ Energy-source nutrients } \\
\hline Energy (kcal) & $0.79(0.4 I-I .54)$ & $0.86(0.4 I-I .8 I)$ & $0.92(0.43-1.99)$ & $1.43(0.67-3.05)$ & 0.51 \\
\hline Protein (g) & $0.7 \mid(0.33-I .49)$ & $0.99(0.4 \mathrm{I}-2.37)$ & $0.84(0.27-2.57)$ & $0.90(0.23-3.49)$ & 0.92 \\
\hline Fat (g) & $0.52(0.23-1.14)$ & $1.05(0.50-2.23)$ & I.I3 (0.46-2.77) & $1.07(0.36-3.20)$ & 0.65 \\
\hline Carbohydrate (g) & $0.42(0.18-1.01)$ & $0.97(0.38-2.52)$ & $0.67(0.17-2.63)$ & I. $15(0.22-6.09)$ & 0.99 \\
\hline Fiber $(\mathrm{g})$ & $0.72(0.32-1.61)$ & $1.05(0.47-2.34)$ & $1.47(0.67-3.23)$ & $1.42(0.57-3.56)$ & 0.21 \\
\hline \multicolumn{6}{|l|}{ Antioxidant vitamins } \\
\hline Vitamin A (RE) & $0.76(0.34-1.68)$ & I.22 (0.57-2.62) & I. $12(0.5 \mathrm{I}-2.48)$ & $1.56(0.7 \mathrm{I}-3.43)$ & 0.20 \\
\hline Retinol $(\mu \mathrm{g})$ & $0.54(0.22-1.29)$ & $1.29(0.64-2.59)$ & $1.04(0.50-2.18)$ & $0.97(0.39-2.22)$ & 0.73 \\
\hline$\beta$-carotene $(\mu \mathrm{g})$ & $0.53(0.23-1.24)$ & $0.87(0.40-1.88)$ & $1.12(0.54-2.33)$ & I. $16(0.55-2.47)$ & 0.36 \\
\hline Vitamin C (mg) & $0.52(0.22-1.23)$ & I.I5 (0.57-2.34) & $0.90(0.4 I-I .96)$ & $0.78(0.33-1.85)$ & 0.92 \\
\hline Folate $(\mu \mathrm{g})$ & $0.78(0.36-1.70)$ & $0.67(0.28-1.6 \mathrm{I})$ & $1.93(0.92-4.05)$ & $\mathrm{I} .38(0.57-3.3 \mathrm{I})$ & 0.14 \\
\hline Vitamin E (mg) & $0.82(0.40-1.67)$ & $0.94(0.42-2.09)$ & $0.92(0.39-2.20)$ & $0.43(0.14-1.38)$ & 0.37 \\
\hline
\end{tabular}

Notes: QI is taken as reference; OR after adjusting for age, sex, marriage status, BMI, history of asthma and tuberculosis, energy, and cigarette smoking.

Abbreviations: OR, odds ratio; $\mathrm{Q}$, quintile; RE, retinol equivalents; BMI, body mass index. 
Table 5 Association of $\mathrm{FEV}_{1}, \mathrm{FVC}$, and $\mathrm{FEV}, / F V C$ with increased intake of vitamins $\mathrm{C}$ and $\mathrm{E}$

\begin{tabular}{|c|c|c|c|c|}
\hline & \multicolumn{2}{|c|}{ Value per increase $100 \mathrm{mg}$ vitamin $\mathrm{C}(\mathrm{mL})$} & \multicolumn{2}{|c|}{ Value per increase $5 \mathrm{mg}$ vitamin $\mathrm{E}(\mathrm{mL})$} \\
\hline & Crude & Adjusted & Crude & Adjusted \\
\hline $\mathrm{FEV}_{\text {, }}$ & $27.2(P=0.03)$ & I7.I $(P=0.03)$ & $14.9(P<0.01)$ & $1.64(P=0.02)$ \\
\hline FVC & $22.2(P=0.15)$ & $24.1(P=0.01)$ & $17.3(P<0.01)$ & $2.01 \quad(P=0.03)$ \\
\hline $\mathrm{FEV}_{\mathrm{I}} / \mathrm{FVC}$ & $2.50(P=0.01)$ & $-0.60(P=0.5 \mathrm{I})$ & $0.24(P=0.0 \mathrm{I})$ & $0.01 \quad(P=0.88)$ \\
\hline
\end{tabular}

Note: The values are adjusted for age, sex, marriage status, BMI, history of asthma and tuberculosis, and cigarette smoking.

Abbreviations: $\mathrm{FEV}_{1}$, forced expiratory volume; FVC, forced vital capacity; BMI, body mass index.

this study. For the socioeconomic factors, lower educational level and household income were associated with the higher risk of COPD as reported by Yin et $\mathrm{al}^{22}$ which suggested that educated and rich people are more likely to have healthy lifestyle and healthy dietary pattern. Consistent with other studies, increased risk of COPD was seen in subjects with history of tuberculosis ${ }^{23,24}$ and asthma in our study. Hnizdo et $\mathrm{al}^{25}$ showed that pulmonary tuberculosis can lead to scarring and accelerated decline in lung function. Eisner et $\mathrm{al}^{26}$ reviewed an evident association between chronic asthma and both airway obstruction and accelerated loss of pulmonary function, and suggested that asthma can predispose a person to develop COPD, as airway obstruction can directly lead to COPD. BMI has been associated with decline in lung function, ${ }^{27,28}$ consistent with the result from our study. The possible explanation for this association could be because of the decrease in cell-mediated immunity and reduction in circulating T-lymphocyte numbers, as a result of protein-energy malnutrition, which can cause increased susceptibility to infections. ${ }^{29}$

Although the mechanism through which vitamin $\mathrm{C}$ plays a preventive role in COPD is not well understood, we can correlate the action mechanism with the valuable properties of vitamin $\mathrm{C}$ in the lungs. Vitamin $\mathrm{C}$ may help in repairing the lung tissue by the synthesis of collagen ${ }^{30}$ and preventing free-radical-induced lipid peroxidation; ${ }^{31}$ in addition, vitamin $\mathrm{C}$ scavenges superoxide radicals and protects against oxidative damage, ${ }^{5}$ thus implying protective role of vitamin $\mathrm{C}$ in lung tissues. We observed that higher intake of vitamin $\mathrm{C}$ was significantly associated with decreased risk of COPD, which is similar to the finding of other study. ${ }^{7}$ The risk of COPD was low in men who belonged to the highest quintile of vitamin $\mathrm{C}$ intake in comparison to the lowest quintile. Several studies reported the fact that the intake of antioxidant vitamin $\mathrm{C}$ may exert an effect on lung function. ${ }^{32,33}$ In our findings, an increase in vitamin $\mathrm{C}$ intake of $100 \mathrm{mg} / \mathrm{d}$ was significantly associated with increase in $\mathrm{FEV}_{1}$ and $\mathrm{FVC}$, which is consistent with the finding of several studies. ${ }^{7,30,34}$ Our findings could provide further evidence that vitamin $\mathrm{C}$ intake is beneficially related to both $\mathrm{FEV}_{1}$ and $\mathrm{FVC}$.

Consistent with a previous study, ${ }^{35}$ higher intake of retinol was associated with the decreased risk of COPD. No significant association was seen between $\beta$-carotene intake and the COPD risk, which elucidates nonbeneficial effect of $\beta$-carotene in human lung as reported by some observational studies. ${ }^{36,37}$ The higher intake of vitamin E was significantly associated with decreased risk of COPD in our study, similar to the finding from other studies. ${ }^{5,8}$ In addition, a significant association was observed between the dietary intake of vitamin $\mathrm{E}$ and $\mathrm{FEV}_{1}$ and $\mathrm{FVC}$ index, supported by other previous reports; ${ }^{34,38}$ however, one study did not show the association. ${ }^{37}$ Oxidative stress is involved in the pathophysiology of reduced pulmonary function and

Table 6 Interaction between smoking and antioxidant vitamins C or E intake on COPD risk among men

\begin{tabular}{|c|c|c|c|c|c|c|}
\hline & \multicolumn{2}{|c|}{ Subjects at risk } & \multicolumn{2}{|c|}{ COPD cases } & \multicolumn{2}{|l|}{ ORs } \\
\hline & Highest & $\overline{\text { Lowest }}$ & Highest & $\overline{\text { Lowest }}$ & Highest & Lowest \\
\hline \multicolumn{7}{|l|}{ Vitamin C } \\
\hline Noncurrent smoker & 1,082 & 475 & 59 & 47 & Ref & $\mathrm{I} .42(0.90-2.24)$ \\
\hline \multirow[t]{2}{*}{ Current smoker } & 835 & 445 & 78 & 71 & $2.54(1.51-4.26)$ & $2.96(1.88-4.67)$ \\
\hline & & & & & & $P_{\text {interaction }}=0.43$ \\
\hline \multicolumn{7}{|l|}{ Vitamin E } \\
\hline Noncurrent smoker & 1,050 & 507 & 54 & 52 & Ref & $1.54(0.94-2.52)$ \\
\hline \multirow[t]{2}{*}{ Current smoker } & 862 & 408 & 87 & 62 & $3.14(1.87-5.25)$ & $2.98(1.78-4.97)$ \\
\hline & & & & & & $P_{\text {interaction }}=0.11$ \\
\hline
\end{tabular}

Note: OR after adjusting for age, marriage status, BMI, history of asthma and tuberculosis, and energy. Abbreviations: OR, odds ratio; BMI, body mass index. 
COPD development. ${ }^{39}$ The in vitro results of Howard et $\mathrm{al}^{40}$ showed that vitamin $\mathrm{E}$ promotes membrane repair from oxidative stress and prevents oxidative-stress-induced failure. In addition, vitamin $\mathrm{E}$ converts ROS into less active forms and acts as chain-breaking antioxidants in lipid peroxidation, ${ }^{41}$ which protects against cell membrane injury. ${ }^{42}$

Smoking activates inflammatory cells in the lungs leading to the production of ROS. Increased level of ROS triggers several cellular processes, like activation of $\mathrm{NF} \kappa \mathrm{B}$, which results in inflammation in patients with COPD. ${ }^{3} \mathrm{~A}$ recent US Surgeon General's report has reviewed smoking and COPD extensively, where $80 \%-90 \%$ of COPD is attributed to smoking in the USA. ${ }^{43}$ Despite the review by Eisner et $\mathrm{al}^{26}$ highlighting untraditional risk factors for COPD rather than solely smoking, studies conducted in Europe and Asia have consistently shown an association between smoking and COPD risk in cross-sectional ${ }^{44,45}$ and in cohort studies. ${ }^{46,47}$ According to the literature, the dietary antioxidants may inhibit cigarette-oxidant-induced NFKB activation, histone acetylation, and proinflammatory cytokine release for the restoration of glucocorticoid functions through upregulation of histone deacetylase activity. ${ }^{3}$ As the percentage of female smokers was very low $(2.2 \%)$ compared to male smokers $(22.89 \%)$, we analyzed the interactive effect between smoking and vitamin $\mathrm{C}$ or $\mathrm{E}$ intake on COPD risk among men. In our study, the risk of COPD for current smokers with the lowest vitamins $\mathrm{C}$ and $\mathrm{E}$ intake was increased almost threefold than noncurrent smokers with high intake of vitamins $\mathrm{C}$ and $\mathrm{E}$. On the other hand, after simple stratification by smoking, we did not find any association between intake of antioxidants and COPD risk (data not shown). To the best of our knowledge, our study is the first study to compare the interactive effects of antioxidants and smoking on COPD risk, although no statistically significant interactions were observed between smoking and vitamin $\mathrm{C}$ or $\mathrm{E}$ intake with COPD risk among men.

Our study has several limitations. First, our definition of COPD (prebronchodilator $\mathrm{FEV}_{1} / \mathrm{FVC}<0.70$ ) represents a simplified case definition for epidemiological purposes and not a definitive clinical diagnosis; however, because of the large sample size, it becomes costly and irrelevant to perform clinical diagnosis of the study subjects. Nondifferential misclassification of COPD diagnosis between case and noncases might underestimate associations between antioxidants intake and COPD risk or lung function; however, our study indicates the presence of an effect, and an estimate of effect without the misclassification would generally be even greater. Second, we used FFQ that depended on the subjects' memories for the dietary intake and they may have had difficulty recalling frequency and food portion size accurately. To overcome this limitation, the use of a closed format and the provision to make choices with food pictures might have made it easier to recall the frequency and portion of their diet. Third, even though this being prospective study, we did not use hazard Cox model because of the relatively short follow-up time (only two times). Nevertheless, a previously published paper reported that the pooled logistic model is useful for the short follow-up time cohort study ${ }^{48}$ and also one published paper showed that pooled logistic regression is close to the time-dependent covariate Cox regression analysis. ${ }^{49}$ Fourth, ingredient database for vitamin $\mathrm{E}$ was not available during the validation study of FFQ and also the validity correlation coefficient for nutrients, cited in the validity of FFQ, was low. Koreans have a unique way of serving and eating foods. A meal is typically comprised of a bowl of rice with mixed soup and multiple side dishes, and the side dishes are shared. So, low perception of proportion size and difficulty in answering the frequency of consumption may be the reason for lower correlation. In addition, the lower interindividual variations in nutrients intakes as reported by other Korean studies ${ }^{50,51}$ may be another reason for lower correlation; however, we can compare the association only when strong relationships between dietary exposure and disease will be detected. Beside these limitations, our study has several strengths too. First, the use of data from a large prospective cohort benefits for the establishment of temporal relationship and certainty about the cause-effect relationship. Second, we included most well-known confounding factors in the analysis such as age, sex, marriage status, BMI, history of asthma and tuberculosis, and cigarette smoking, because of large sample size. Third, FFQ was developed based on nationwide dietary data and hence the use of validated FFQ strengthened the reproducibility of our results.

In conclusion, this prospective study suggests that antioxidants vitamins, particularly vitamins $\mathrm{C}$ and $\mathrm{E}$, have a beneficial effect on the risk of COPD and lung function $\left(\mathrm{FEV}_{1}\right.$ and $\mathrm{FVC}$ ). In addition, the antioxidant effects of vitamins $\mathrm{C}$ and $\mathrm{E}$ on the risk of COPD might be stronger in men who smoke than in noncurrent smokers.

\section{Acknowledgment}

We thank all the staff members involved in each study area for their valuable efforts in conducting the baseline surveys. This study was supported by the Ministry of Environment, Republic of Korea, and by the Department of Internal 
Medicine and Environmental Health Center, Kangwon National University.

\section{Disclosure}

The authors report no conflicts of interest in this work.

\section{References}

1. Pauwels RA, Buist AS, Calverley PM, Jenkins CR, Hurd SS. Global strategy for the diagnosis, management, and prevention of chronic obstructive pulmonary disease. NHLBI/WHO Global Initiative for Chronic Obstructive Lung Disease (GOLD) Workshop summary. Am J Respir Crit Care Med. 2001;163(5):1256-1276.

2. Tan WC, Seale P, Ip M, et al. Trends in COPD mortality and hospitalizations in countries and regions of Asia-Pacific. Respirology. 2009;14(1): 90-97.

3. Rahman I. Antioxidant therapies in COPD. Int J Chron Obstruct Pulmon Dis. 2006;1(1):15-29.

4. Heffner JE, Repine JE. Pulmonary strategies of antioxidant defense. Am Rev Respir Dis. 1989;140(2):531-554.

5. Hanson C, Rutten EP, Wouters EF, Rennard S. Diet and vitamin D as risk factors for lung impairment and COPD. Transl Res. 2013;162(4): 219-236.

6. Varraso R, Fung TT, Hu FB, Willett W, Camargo CA. Prospective study of dietary patterns and chronic obstructive pulmonary disease among US men. Thorax. 2007;62(9):786-791.

7. Schwartz J, Weiss ST. Relationship between dietary vitamin C intake and pulmonary function in the First National Health and Nutrition Examination Survey (NHANES I). Am J Clin Nutr. 1994;59(1):110-114.

8. Walda IC, Tabak C, Smit HA, et al. Diet and 20-year chronic obstructive pulmonary disease mortality in middle-aged men from three European countries. Eur J Clin Nutr. 2002;56(7):638-643.

9. $\mathrm{Hu}$ G, Cassano PA. Antioxidant nutrients and pulmonary function: the Third National Health and Nutrition Examination Survey (NHANES III). Am J Epidemiol. 2000;151(10):975-981.

10. Keranis E, Makris D, Rodopoulou P, et al. Impact of dietary shift to higher-antioxidant foods in COPD: a randomised trial. Eur Respir J. 2010;36(4):774-780.

11. Tsiligianni IG, van der Molen T. A systematic review of the role of vitamin insufficiencies and supplementation in COPD. Respir Res. 2010; $11: 171$.

12. Smit HA, Grievink L, Tabak C. Dietary influences on chronic obstructive lung disease and asthma: a review of the epidemiological evidence. Proc Nutr Soc. 1999;58(2):309-319.

13. Tabak C, Smit HA, Heederik D, Ocke MC, Kromhout D. Diet and chronic obstructive pulmonary disease: independent beneficial effects of fruits, whole grains, and alcohol (the MORGEN study). Clin Exp Allergy. 2001;31(5):747-755.

14. Shin C, Abbott RD, Lee H, Kim J, Kimm K. Prevalence and correlates of orthostatic hypotension in middle-aged men and women in Korea: the Korean Health and Genome Study. J Hum Hypertens. 2004;18(10): 717-723.

15. Ahn Y, Kwon E, Shim JE, et al. Validation and reproducibility of food frequency questionnaire for Korean genome epidemiologic study. Eur J Clin Nutr. 2007;61(12):1435-1441.

16. Ahn Y, Lee J, Paik HY, Lee HK, Jo I, Kimm K. Development of a semi-quantitative food frequency questionnaire based on dietary data from the Korean National Health and Nutrition Examination Survey. Nutr Sci. 2003;6(3):173-184.

17. Han MK, Postma D, Mannino DM, et al. Gender and chronic obstructive pulmonary disease: why it matters. Am J Respir Crit Care Med. 2007; 176(12):1179-1184.

18. Thyagarajan B, Jacobs DR Jr, Apostol GG, et al. Longitudinal association of body mass index with lung function: the CARDIA study. Respir Res. 2008;9:31.
19. Vestbo J, Prescott E, Almdal T, et al. Body mass, fat-free body mass, and prognosis in patients with chronic obstructive pulmonary disease from a random population sample: findings from the Copenhagen City Heart Study. Am J Respir Crit Care Med. 2006;173(1):79-83.

20. Wang KS, Wang L, Zheng S, Wu LY. Associations of smoking status and serious psychological distress with chronic obstructive pulmonary disease. Int J High Risk Behav Addict. 2013;2(2):59-65.

21. Loganathan RS, Stover DE, Shi W, Venkatraman E. Prevalence of COPD in women compared to men around the time of diagnosis of primary lung cancer. Chest. 2006;129(5):1305-1312.

22. Yin P, Zhang M, Li Y, Jiang Y, Zhao W. Prevalence of COPD and its association with socioeconomic status in China: findings from China Chronic Disease Risk Factor Surveillance 2007. BMC Public Health 2011;11:586.

23. Humbert M. The burden of pulmonary hypertension. Eur Respir J. 2007;30(1):1-2.

24. Caballero A, Torres-Duque CA, Jaramillo C, et al. Prevalence of COPD in five Colombian cities situated at low, medium, and high altitude (PREPOCOL study). Chest. 2008;133(2):343-349.

25. Hnizdo E, Singh T, Churchyard G. Chronic pulmonary function impairment caused by initial and recurrent pulmonary tuberculosis following treatment. Thorax. 2000;55(1):32-38.

26. Eisner MD, Anthonisen N, Coultas D, et al. An official American Thoracic Society public policy statement: novel risk factors and the global burden of chronic obstructive pulmonary disease. Am J Respir Crit Care Med. 2010;182(5):693-718.

27. Harik-Khan RI, Fleg JL, Wise RA. Body mass index and the risk of COPD. Chest. 2002;121(2):370-376.

28. Ubilla C, Bustos P, Amigo H, Oyarzun M, Rona RJ. Nutritional status, especially body mass index, from birth to adulthood and lung function in young adulthood. Ann Hum Biol. 2008;35(3):322-333.

29. Chandra RK. Cell-mediated immunity in nutritional imbalance. Fed Proc. 1980;39(13):3088-3092.

30. Hu G, Zhang X, Chen J, Peto R, Campbell TC, Cassano PA. Dietary vitamin $\mathrm{C}$ intake and lung function in rural China. Am J Epidemiol. 1998;148(6):594-599.

31. Sies H, Stahl W. Vitamins E and C, beta-carotene, and other carotenoids as antioxidants. Am J Clin Nutr. 1995;62(6 Suppl):1315S-1321S.

32. McKeever TM, Scrivener S, Broadfield E, Jones Z, Britton J, Lewis SA. Prospective study of diet and decline in lung function in a general population. Am J Respir Crit Care Med. 2002;165(9):1299-1303.

33. Ochs-Balcom HM, Grant BJ, Muti P, et al. Antioxidants, oxidative stress, and pulmonary function in individuals diagnosed with asthma or COPD. Eur J Clin Nutr. 2006;60(8):991-999.

34. Schunemann HJ, McCann S, Grant BJ, Trevisan M, Muti P, Freudenheim JL. Lung function in relation to intake of carotenoids and other antioxidant vitamins in a population-based study. Am J Epidemiol. 2002;155(5):463-471.

35. Morabia A, Sorenson A, Kumanyika SK, Abbey H, Cohen BH, Chee E. Vitamin A, cigarette smoking, and airway obstruction. Am Rev Respir Dis. 1989;140(5):1312-1316.

36. Soutar A, Seaton A, Brown K. Bronchial reactivity and dietary antioxidants. Thorax. 1997;52(2):166-170.

37. Grievink L, Smit HA, Ocke MC, van 't Veer P, Kromhout D. Dietary intake of antioxidant (pro)-vitamins, respiratory symptoms and pulmonary function: the MORGEN study. Thorax. 1998;53(3):166-171.

38. Tabak C, Smit HA, Rasanen L, et al. Dietary factors and pulmonary function: a cross sectional study in middle aged men from three European countries. Thorax. 1999;54(11):1021-1026.

39. Yao H, Rahman I. Current concepts on oxidative/carbonyl stress, inflammation and epigenetics in pathogenesis of chronic obstructive pulmonary disease. Toxicol Appl Pharmacol. 2011;254(2):72-85.

40. Howard AC, McNeil AK, McNeil PL. Promotion of plasma membrane repair by vitamin E. Nat Commun. 2011;2:597.

41. Burton GW, Joyce A, Ingold KU. First proof that vitamin $\mathrm{E}$ is major lipid-soluble, chain-breaking antioxidant in human blood plasma. Lancet. 1982;2(8293):327. 
42. Burton GW, Joyce A, Ingold KU. Is vitamin E the only lipid-soluble, chain-breaking antioxidant in human blood plasma and erythrocyte membranes? Arch Biochem Biophys. 1983;221(1):281-290.

43. US Department of Health and Human Services. The Health Consequences of Smoking-50 Years of Progress: A Report of the Surgeon General. Atlanta, GA: US Department of Health and Human Services; 2014.

44. Jaen Diaz JI, de Castro Mesa C, Gontan Garcia-Salamanca MJ, Lopez de Castro F. Prevalence of chronic obstructive pulmonary disease and risk factors in smokers and ex-smokers. Arch Bronconeumol. 2003;39(12): 554-558.

45. Kurmi OP, Li L, Wang J, et al. COPD and its association with smoking in the Mainland China: a cross-sectional analysis of 0.5 million men and women from ten diverse areas. Int J Chron Obstruct Pulmon Dis. 2015;10:655-665.

46. Lindberg A, Jonsson AC, Ronmark E, Lundgren R, Larsson LG, Lundback B. Ten-year cumulative incidence of COPD and risk factors for incident disease in a symptomatic cohort. Chest. 2005;127(5): 1544-1552.
47. Lokke A, Lange P, Scharling H, Fabricius P, Vestbo J. Developing COPD: a 25 year follow up study of the general population. Thorax. 2006; 61(11):935-939.

48. Baik I, Abbott RD, Curb JD, Shin C. Intake of fish and n-3 fatty acids and future risk of metabolic syndrome. J Am Diet Assoc. 2010;110(7): 1018-1026.

49. D’Agostino RB, Lee ML, Belanger AJ, Cupples LA, Anderson K, Kannel WB. Relation of pooled logistic regression to time dependent Cox regression analysis: the Framingham Heart Study. Stat Med. 1990; 9(12):1501-1515.

50. Kwon E, Ahn Y, Shim JE, et al. Within- and between individual variation in nutrient intakes with day of the week and season in Korean adults. Korean J Nutr. 2004;37(10):917-927.

51. Oh SY, Lee H, Paik HY. Within- and between-individual variation in nutrient intakes assessed by recall and record methods among college women. Korean J Nutr. 1996;29(9):1028-1034.

\section{Publish your work in this journal}

The International Journal of COPD is an international, peer-reviewed journal of therapeutics and pharmacology focusing on concise rapid reporting of clinical studies and reviews in COPD. Special focus is given to the pathophysiological processes underlying the disease, intervention programs, patient focused education, and self management protocols.

\section{Dovepress}

This journal is indexed on PubMed Central, MedLine and CAS. The manuscript management system is completely online and includes a very quick and fair peer-review system, which is all easy to use. Visit $\mathrm{http}: / / \mathrm{www}$. dovepress.com/testimonials.php to read real quotes from published authors. 